\title{
Drunk driving prevention and cultural influences: the SAFE ROADS 4 YOUTH (SR4Y) project
}

\author{
Dr. Jean-PascalAssailly \& Dr. Julien Cestac \\ IFSTTAR, Allée des marroniers, 78000, Satory, France
}

\begin{abstract}
Driving under the influence of alcohol is an important factor in road fatalities all over the world. However, an important significant heterogeneity among countries was found regarding this issue. Why is such a discrepancy found among countries? Several specificities might explain the differences, such as enforcement practices, cultural values, or drinking patterns. The aim of this study was to test whether the social influence (descriptive and injunctive norms) on drunk driving is country-dependent and to explore whether the differences can be attributed to the contextual and/or cultural specificities of the countries.
\end{abstract}

We used data from the SAFE ROADS 4 YOUTH (SR4Y) project, with the objective to implement and evaluate drink driving prevention actions using a common conceptual framework in three developing countries over three continents: Vietnam, South Africa and Argentina. 11300 students aged between 13 and 25 were surveyed.

We conducted a regression analysis of social norms on the intention to resist to drunk driving in each country. In all three countries, family injunctive norms were correlated with drunk-driving (DD) intention. The link was strongest in Argentina and weakest in South Africa. In South Africa, friends' injunctive norms had negligible link with DD intention, no link was found with friends' descriptive norms. The link between injunctive norms and DD intention was stronger in Vietnam than in Argentina. Friends' descriptive norms had stronger link with $D D$ in Argentina than in Vietnam.

Overall these results suggest differences in social influence on drunk driving intention depending on national cultures.

KEYWORDS: Alcohol; culture; young people; drinking and driving; risk perception; norms

\section{INTRODUCTION}

All over the world, drink-driving is an important factor in road fatalities. However, some differences between countries were found regarding this issue. In 2010, the share of road fatalities linked to drink-driving was lower than $10 \%$ in many countries such as Japan, Austria, Germany or the Netherlands whereas it was higher than $30 \%$ in many other countries such as France, Greece, Spain, Canada or the USA (International Transport Forum, 2018). Why was such a discrepancy found among countries? Several country-specific factors (enforcement, alcohol consumption or cultural values) might explain these differences (Cestac, Kraïem \& Assailly, 2016). According to the Lederman's Law (1956): the level of alcohol-related harm in a country, such as cirrhosis, fights, traffic crashes, etc. is correlated to the yearly average consumption of the population. Moreover, the Driving Under the Influence of Drugs (DRUID) project conducted in 13 European countries (Houwing et al., 2011) found that an average of $3.48 \%$ of all European drivers on the road have alcohol in their blood (varying from $0.15 \%$ of drivers in Hungary to $8.59 \%$ in Italy). These frequencies of drink-driving have of course a great impact on alcohol-related road fatalities, but why some countries have more drunk drivers than do others?

One of the factors that may predict drunk driving is social influence (Fernandes et al., 2010). In several countries, it has been observed that risky behaviors may be influenced by peers, we may therefore consider it as a "universal" process. Though the relation between peers' and individuals' behaviors has been moderated by cultural values in some studies (Gazis et al., 2009). The discrepancy between countries regarding alcohol-related fatalities may thus be linked to differences in the level of peer influences on drunk driving (Cestac, Kraïem \& Assailly, 2016). Moreover, the driving context (traffic laws, infrastructures, en- 
forcement levels, etc.) depends on the country and these contextual factors may also influence drunk driving and moderate peers' influences on this behavior. The social context may influence behaviors, and for example alcohol use and driving behavior are particularly sensitive to others' expectations and behaviors. The social norms, either descriptive or injunctive (see Deutsch and Gerard, 1955), have been often studied in their links with risk-taking at the wheel. For example, Forward (2009) showed that speeding and dangerous overtaking were influenced by descriptive norms. More recently, the interplay of descriptive and injunctive norms on the speeding intentions among young French drivers have been observed (Cestac, Paran \& Delhomme, 2014). Alcohol use (Larimer et al., 2004) and impaired driving (Brown, 1998; Fernandes et al., 2010) are also related to peers' drinking behaviors and drunk driving respectively.

If the relation between personal behaviors and friends' behaviors has often been analyzed as a social influence, it has also been argued that it could be the consequence of a selection bias, that is, people selecting as friends others who behave like them (Curran et al., 1997). Nevertheless, the desire for conformity is linked with the motivation to be integrated in the group and is reinforced by the fear of social sanction. It is thus possible that socialization and selection operate simultaneously. Still, Stok and colleagues (2014) observed a direct influence of descriptive norms on behavior in an experimental setting.

Other important determinants of social norms are individual beliefs about risk and safety. These beliefs may be false and induce erroneous attitudes and behaviors. False beliefs can be the consequence of ignorance about actual risks or of perception biases. They can be transmitted through peer and/or parental influence and are sensitive to the cultural context (Assailly, 2011).

\section{Cultural effects}

Culture has been described as a collective state of mind shared among members of a specific population (Hofstede, 2001). It covers norms, values, and beliefs that varies among subgroups of populations. Differences between driving styles in countries or groups of countries have been identified by cross-cultural researchers. Driving behaviors have been compared in six European and Middle Eastern countries: in Western/Northern European countries slightly more ordinary violations are observed (i.e., speeding on a mo- torway) but less aggressive violations (get angry, give chase) and errors (e.g., nearly hit cyclist while turning right) than in Southern European and Middle Eastern countries (Özkan et al., 2006). The relationship between the culture and the number of accidents is also under the influence or these driving behaviors. These differences were again observed in a more recent comparison of Northern European countries (i.e., Finland and Sweden) and Southern European countries (i.e., Greece and Turkey), and significant differences in drunk driving across these countries have been recorded (Warner et al., 2011). Though, differences between Southern European countries also exist. For example, Italian and Greek students' risky behaviors have been compared: Italian students comply more with safety measures but report more drunk-driving behaviors (Antonopoulos et al., 2011).

We thus have decided to study social influence on drink driving intention among young students with a cross-cultural perspective. Cultural values and social norms can be considered as two kinds of norms. Peer influence is the consequence of a social norm at the individual level (what significant others are expecting or doing). More general normative influences such as national cultures may also have an impact at the individual level. Some moral norms are transmitted by cultural values (what is morally acceptable in the country), and some legal norms belong to the driving context in a country (driving laws, infrastructures, or education). These norms may vary across individuals and situations and therefore may be conflictual (Engel, 2007).

If social influences on drunk driving has been well studied, cultural effects are less well known. Crosscultural research on driving behavior has compared different countries about their driving style but has given much less explanations about how a culture may influence drunk driving (Antonopoulos et al., 2011; Özkan et al., 2006; Warner et al., 2011). One objective of this study is to see the ways in which culture and national contexts affect drunk driving, in particular the ways in which they affect social influence. Another determinant of violations is one dimension of risk perception which is false beliefs. These misperceptions about alcohol use (for example, "coffee decreases the blood alcohol concentration") or speed (for example, "speed is not the cause of crashes, I can drive fast safely") may lead to a dangerous driving style. These misperceptions may be under the influence of cultural or contextual factors (for example, lack of education or communication on these risk factors). 


\section{RESEARCH QUESTIONS AND HYPOTHESES}

The objective of this study is to see whether the social influence on drunk driving is dependent of the country and to analyze if the differences can be attributed to the contextual and/or cultural specificities of the countries. More specifically, we have proposed two general hypotheses guiding our work.

First, as demonstrated by Ando et al. (2007) and Cestac et al. (2016), the strength of the descriptive norm's influence on one's behavior may vary across cultures. We thus expect that the link between friends' expectations and behaviors and respondents' behaviors will be stronger in some countries than in others. Indeed, normative influence, in particular injunctive norms influence on behaviors, is stronger in collectivistic countries where the interdependence between society members is high. We thus assume that this influence will be the greatest in Vietnam and the lowest in South Africa (Hypothese 1).

Second, we expect that false beliefs about alcohol use and about drunk driving will vary across cultures. Moreover, it seems logical to expect higher risk taking and crashes in countries with more false beliefs (Hypothese 2).

\section{METHOD}

The SAFE ROADS 4 YOUTH (SR4Y) project is an international project, led from 2012 to 2016, with the objective to implement and evaluate drink driv- ing prevention actions using a common conceptual framework in three developing countries over three continents: Vietnam, South Africa and Argentina. It aimed to determine what types of community programs are most effective in preventing drink driving and how their effect varies across countries, traffic policy contexts and cultures. The project had a very large scope including before/after measures with prevention interventions in some schools and some control groups. Moreover, in some countries the project was also interested in professional drivers. However in the present paper we focus our analyses on a smaller part of the data: students aged between 13 and 25 and only for the first wave of measures (i.e., before any specific intervention) with $N=11300$. Detailed characteristics of each national convenience sample can be found in Table 1 . We observed differences between the samples regarding mobility patterns with much lower overall motorized trips in South Africa and more moped drivers in Vietnam, $\chi^{2}(2,11496)=223, p<.001$. There were also differences in overall alcohol consumption which is much higher in Argentina, and regarding the gender gap in alcohol consumption which is very large in Vietnam.

The three countries involved in the project can be compared using existing data (see Table 2) from Hofstede, Hofstede \& Minkov (2010) ${ }^{1}$. Argentina and South Africa appear to be relatively close to each other regarding these dimensions compared to Vietnam. However they differ in Individualism which is greater in South Africa and in Uncertainty avoidance

Table 1: Descriptive statistics of national samples

\begin{tabular}{lccc}
\hline & Argentina & South Africa & Vietnam \\
\hline $\mathbf{N}$ & 5448 & 1033 & 4819 \\
M Age (SD) & $17.6(2.4)$ & $17.6(2.1)$ & $18.1(2.8)$ \\
\% Women & 56.8 & 58.2 & 52.6 \\
\% moped drivers & 6.9 & 3.8 & 14.4 \\
\% motorcyclists drivers & 29.6 & 6.9 & 23.7 \\
\% car drivers & 22.5 & 18.2 & 2.4 \\
\% of alcohol abstainers. Total & 14.3 & 53.3 & 60.6 \\
\% of alcohol abstainers. Females & 15.9 & 53 & 78.3 \\
\% of alcohol abstainers. Males & 12.2 & 54 & 41.4 \\
\hline
\end{tabular}


which score is higher for Argentina. The high level of Uncertainty avoidance in Argentina lead to a very dense legislation including a large number of specific laws, sometimes contradictory. In Vietnam, Power distance is very high, which means that hierarchical order and inequalities are well accepted by the population, more than in the two other countries involved in the present study. Vietnam is also clearly a collectivistic country, meaning that people feel a high responsibility for other members of their groups and that offences often lead to shame.

Table 2: Country scores on Hofstede's (2010) dimensions

\begin{tabular}{lccc}
\hline & Argentina & $\begin{array}{c}\text { South } \\
\text { Africa }\end{array}$ & Vietnam \\
\hline Power distance & 49 & 49 & 70 \\
Individualism & 46 & 65 & 20 \\
Masculinity & 56 & 63 & 40 \\
Uncertainty avoidance & 86 & 49 & 30 \\
Long term orientation & 20 & 34 & 57 \\
Indulgence & 62 & 63 & 35 \\
\hline
\end{tabular}

The measures analyzed in the present study are detailed below. For the first four variables answers were collected using 5-points Likert scales ranging from 1 (strongly disagree) to 5 (strongly agree). For the beliefs questions answers were "True", "False", "I don't know".

Table 3: Reported behaviors and beliefs

\begin{tabular}{lccccc}
\hline & Argentina & South Africa & Vietnam & F & eta $^{2}$ \\
\hline Average number of crashes in the last year & 0.39 & 0.60 & 1.13 & $189 * * *$ \\
False beliefs (out of 12) & 3.56 & 4.54 & 4.23 & $259 * * *$ & .03 \\
\hline
\end{tabular}

Note: $* * *=\mathrm{p}<.001$

Table 4: Regression of social norms on the intention to resist to drunk driving

\begin{tabular}{lccc}
\hline & $\begin{array}{c}\boldsymbol{\beta} \\
\text { South Africa } \\
\text { adj. } R^{2}=.02\end{array}$ & $\begin{array}{c}\boldsymbol{\beta} \\
\text { Vietnam } \\
\text { adj. } R^{2}=.08\end{array}$ & $\begin{array}{c}\boldsymbol{\beta} \\
\text { Argentina } \\
\text { adj. } R^{2}=.09\end{array}$ \\
\hline Friend's injunctive norms & $.08^{*}$ & $.20^{* * *}$ & $.15^{* * *}$ \\
Friend's descriptive norms & $\mathrm{ns}$ & $-.04 * *$ & $-.10^{* * *}$ \\
Family injunctive norms & $.10^{* * *}$ & $.14^{* * *}$ & $.21 * * *$ \\
\hline
\end{tabular}

Note: ${ }^{*}=\mathrm{p}<.05, * *=\mathrm{p}<.01, * * *=\mathrm{p}<.001$ 
between the number of false beliefs and number of reported crashes, $\mathrm{F}(2,11235)=17.4, \mathrm{p}<.001$, however the effect size is negligible ( eta $^{2}<.01$ ).

In each country, we conducted a linear regression analysis of social norms on the intention to resist drink driving. All condition indices were lower than 15 , for each country, indicating no collinearity issue. In all three countries, family injunctive norms were linked with drunk-driving (DD) intention. The link was strongest in Argentina and weakest in South Africa. In South Africa, friends' injunctive norms had negligible link with DD intention, no link was found with friends' descriptive norms. The link between friends' injunctive norms and DD intention was stronger in Vietnam than in Argentina. Friends' descriptive norms had stronger link with DD in Argentina than in Vietnam. Overall, based on Cohen's (1988) recommendations for interpretations of effects sizes, we found social norms had a very weak impact on intention to resist drunk driving in South Africa and a moderate link in Vietnam and Argentina.

The three regression models were significantly different from each other. According to the Chow (1960) test, the model for South Africa is different than the model for Vietnam $\mathrm{F}(3,5891)=2013$, $\mathrm{p}<.001$, the model for South Africa is different than the model for Argentina F $(3,6359)=1295, p<.001$, and the model for Argentina is different than the model for Vietnam $F(3,10306)=516, p<.001$.

\section{CONCLUSION AND DISCUSSION}

Concerning our hypotheses on the social influences, both were confirmed: social norms influences can be observed in the three countries, but are not identical and the strength of the influence of descriptive norms on one's behavior vary across cultures.

In the same way, the frequencies of false beliefs are different according to the country; there is a link between number of false beliefs and number of reported crashes, however the effect size is negligible.

The present study suffers from several limitations. Indeed, as a correlational and field study it was not possible to control for any potential confounding variables, as it is always the case for cross-cultural studies. The moderate level of the observed correlations further confirm that other variables are at play when it comes to explain the intention of resisting drink-driving.
Other observations stemming from this project are interesting for the study of cultural factors:

A first and important finding of our work is that amount and frequency of alcohol use among young people has been underestimated in emerging countries. The amount and frequency of alcohol use is increasing nowadays among young people of developing countries precisely because of modernization: enrichment, destabilization of family relationships, pace of social change, rising expectations for academic performance, urbanization and emigration from rural areas, changing gender roles, etc. All these are factors and pressures affecting risk behaviors and alcohol use.

We have seen also from our baseline survey how traffic safety is an important issue among young people in developing countries. The problem is very severe in Vietnam, due to chaotic two-wheel traffic, where half of the subjects had at least one accident in the last three years, compared to $30 \%$ in South Africa and $25 \%$ in Argentina. Due to the two-wheel traffic, accidents are more severe (in terms of injuries) in Vietnam; the economic burden of this safety problem is enormous for this country.

Our survey gives new elements on the well-known issue of gender and risk behaviors. With the comparison of the three countries, we see the influence of culture on gender-related differences, as the gender gap is smaller in South Africa than in Vietnam. For example, in South Africa, $30 \%$ of girls have had traffic accidents in the previous three years, the same proportion as boys, which is a very unusual observation in traffic safety research. No difference was observed concerning the severity of the accidents, which is different than in western countries. $9 \%$ of girls declare drink driving, the same proportion as boys. $26 \%$ had been the passenger of a drunk driver, compared to $20 \%$ of boys. The amount and frequency of alcohol use are very similar between boys and girls, which is not the case in most western countries.

Gender differences are a good example of the relationship between universal and cultural factors: the vulnerability of males concerning alcohol-related accidents and offences has universal biological (effect of hormones), psychological (adherence to gender stereotypes) and anthropological (concern for others and orientation of violence) mechanisms, which are very much the same around the world. However, the historical trend towards the reduction of gender difference varies according to country-specific cultural factors, and the gender equality agenda. Let 
us take as an example the drink driving offense: in some countries where women have no access to car driving or to alcohol, male vulnerability is $100 \%$. Conversely, in the most feminist countries (Scandinavia, Iceland, Australia), the gender gap is reduced to the point of becoming nonexistent. Finally, in southern Europe for instance, gender differences in drink driving are still significant, due to a greater adherence to sex stereotypes.

Concerning the difference between South Africa and Vietnam about alcohol use among boys/girls, another hypothesis could be raised. Since cultural factors, and the descriptive and injunctive norms (what others/parents/ peers will think about me if I drink too much) are stronger in Vietnam than in South Africa, this may put more pressure on Vietnamese girls than on South African ones. This may be understood as a culture/context effect : in Vietnam, the ancient Confucean doctrine still influence the respect of parents and of parents' views of thinking, whereas in South Africa, children are much more "let to themselves", with very few parental supervision. All this is also subject to historical evolutions: the situation was probably different 20 years ago in both countries.

So, in the three countries, the gender differences concerning alcohol use and accidents present an historical evolution and become less important than in the past (impacts of globalization and of the gender equality and women's rights agenda). The more gender equality exists in a country, the smaller are the gender differences in drinking behavior as it gives more legitimacy and fewer stigmas for women to adopt "masculine" behaviors.

Finally, we now have a better understanding of the psychological mechanisms leading to drink driving, and this suggests tracks for the preventive actions to be implemented:

a) tracks from the hypotheses and the results presented above :

- Lack of knowledge and misperceptions: Very large proportions of subjects do not know the legal limit and overestimate the number of drinks to be consumed in one hour to drive legally or safely. Educational actions in various settings may improve this.

- False beliefs leading to overconfidence: Such as "drinking a little alcohol can improve a driver's reflexes”, or “drinking coffee helps to lower the blood alcohol concentration".
False beliefs may be associated to accident involvement.

b) tracks from the general outcomes of the entire SR4Y project :

- Discrepancy between subjective norms: Subjects report that most of their peers would disapprove or even condemn drink driving but that ... most of their peers do it sometimes. Peer-to-peer approaches such as group discussions, role-play, etc are shown to be useful on this topic.

- Positive drinking expectancies: To have fun, release tension, favor group integration or sexual life. This suggests messages for preventive actions: to try to keep the same objectives for the subjects, but with different strategies (ex: how to have fun without or with less alcohol).

- Lack of parental supervision, failure in resisting peer pressure and absence of nighttime trips planning: three important factors of alcohol-related accidents, which have been reported in our survey. Parent-based interventions and "life-skills training" type actions may improve these phenomena.

- Underage drinking and unlicensed driving have been revealed by our survey, when we compare the modes of mobility and the ownership of licenses reported. More generally, fear of detection of underage drinking, or of unlicensed driving or drunk driving, is still very low in the three countries. A capacity building of police enforcement could produce positive effects on these behaviors.

To conclude, differences in social influence on traffic behaviours is a promising field of study and of preventive actions in developing countries, as traffic safety policies have to take into account these specificities.

\section{REFERENCES}

Ando, K., Ohnuma, S., \& Chang, E. C. (2007). Comparing normative influences as determinants of environmentally conscious behaviours between the USA and Japan. Asian Journal Of Social Psychology, 10(3), 171-178.

Antonopoulos, C. N., Germeni, E., Bacopoulou, F., Kalapoki, V., Maltezos, S., Skalkidis, I., Petridou, E. (2011). Assessing the impact of risk-taking behavior on road crash involvement 
among university students residing in two Mediterranean countries. Safety Science, 49,933-938.

Assailly, J.-P. (2011). The psychology of risk. New York: Nova Science, 291 p.

Brown, S. L. (1998). Associations between peer drink driving, peer attitudes toward drink driving, and personal drink driving. Journal Of Applied Social Psychology, 28(5), 423-436.

Cestac, J., Delhomme, P., \& Paran, F. (2014). Drive as I say, not as I do: influence of injunctive and descriptive norms combination on speeding intention among young drivers. Transportation research part F, 23, 44-56. doi: 10.1016/j.trf.2013.12.006.

Cestac, J., Kraïem, S., \& Assailly, J-P. (2016). Cultural values and random breath tests as moderators of the social influence on drunk driving in 15 countries. Journal of Safety Research, 56, 89-96. doi: 10.1016/j.jsr.2015.12.001

Chow, G., 1960. Test of equality between sets of coefficients in two linear regressions. Econometrica, 28 (3), 591-605.

Cohen, J. (1988). Statistical power analysis for the behavioral sciences (2nd ed.). Hillsdale, NJ: Erlbaum.

Curran, P. J., Stice, E., Chassin, L. (1997). The relation between adolescent alcohol use and peer alcohol use: A longitudinal random coefficients model. Journal Of Consulting And Clinical Psychology, 65(1), 130-140.

Deutsch, M., \& Gerard, H. (1955). A study of normative and informational social influences upon individual judgment. The Journal of Abnormal and Social Psychology, 51(3), 629-636. http://dx.doi.org/10.1037/h0046408.

Ecorys (2014). Study on the prevention of drink-driving by the use of alcohol interlock devices. Final Report Rotterdam 18.2.2014.

Engel, R. (2007). Social and cultural factors. TRACE report D5.4. 46 p. Retrieved from http://www.trace-project.org/publication/archives/trace-wp5-d5-4.pdf

Fernandes, R., Hatfield, J., \& Soames Job, R. F. (2010). A systematic investigation of the differential predictors for speeding, drink-driving, driving while fatigued, and not wearing a seat belt, among young drivers. Transportation Research Part F: Traffic Psychology And Behaviour, 13(3), 179-196.

Forward, S. (2009). The theory of planned behaviour: The role of descriptive norms and past behaviour in the prediction of drivers' intentions to violate. Transportation Research Part F: Traffic Psychology and Behaviour, 12(3), 198-207.

Gazis, N., Connor, J. P., Ho, R. (2009). Cultural identity and peer influence as predictors of substance use among culturally diverse Australian adolescents. The Journal Of Early Adolescence, 30(3), 345-368.

Hofstede, G. (2001). Culture's consequences: Comparing values, behaviors, institutions and organizations across nations (2nd ed.). Thousand Oaks, CA: Sage.

Hofstede, G., Hofstede G. J., \& Minkov, M. (2010). Cultures and organizations: Software of the mind. Revised and Expanded 3rd Edition. New York: McGraw-Hill.

Houwing, S., Hagenzieker, M., Mathijssen, R., Bernholf, I. M., Hels, T., Janstrup, K., Verstraete, A. (2011). Prevalence of alcohol and other psychoactive substances in drivers in general traffic. Part I: General Results (Project NoTREN-05-FP6TRS07. 61320-518404-DRUID, Deliverable D2.2.3.). Retrieved from DRUID Reports http://www.druid-project.eu/cln_031/ nn_107534/Druid/EN/deliverales-list/deliverables

International Transport Forum (2018). Alcohol-related road casualties in official crash statistics. Paris: OECD Publications.

Larimer, M., Turner, A., Mallett, K., Geisner, I. (2004). Predicting drinking behavior and alcohol-related problems among fraternity and sorority members: examining the role of descriptive and injunctive norms. Psychology of Addictive Behaviors, 18(3), 203-212.

Özkan, T., Lajunen, T., Chliaoutakis, J. E., Parker, D., Summala (2006). Cross-cultural differences in driving behaviours : a comparison of six countries. Transportation Research Part F, 9,227-242.

Room, R. (2001). Intoxication and bad behavior: understanding cultural differences in the link. Social Science \& Medicine, 53(2), 189-198.

Safe roads for youth (2015). https://www.saferoads4youth.net

Stok, F., Ridder, D. D., Vet, E., Wit, J. F. (2014). Don't tell me what I should do, but what others do: The influence of descriptive and injunctive peer norms on fruit consumption in adolescents. British Journal of Health Psychology, 19(1), 52-6.

Warner, H. W., Özkan, T., Lajunen, T., Tzamalouka, G. (2011). Cross-cultural comparison of drivers' tendency to commit different aberrant driving behaviours. Transportation Research Part F, 14,390-399. 